\title{
Impuestos extrafiscales en la reforma tributaria 2014: análisis crítico*
}

\author{
Patricia Toledo Zúñiga** \\ Francisca Riffo Aguilar*** \\ Pablo Torres Pedreros****
}

\begin{abstract}
RESUMEN
Este trabajo aborda los denominados "impuestos extrafiscales" incorporados con la Reforma Tributaria del 2014 por medio de la Ley $N^{\circ} 20.780$.

La finalidad principal de los impuestos extrafiscales no es obtener ingresos para el Estado, sino modificar externalidades negativas como el daño a la salud y la contaminación medioambiental. El propósito de este trabajo es analizar los impuestos extrafiscales de la Reforma Tributaria 2014 y demostrar que, no obstante su denominación, su finalidad es principalmente recaudatoria.
\end{abstract}

Extrafiscalidad - reforma tributaria 2014 - impuestos

\section{Corrective taxes in the tax reform: critical analysis}

\begin{abstract}
This work addresses the so-called "corrective taxes" scheme established in 2014's Tax Reform by means of the Law Nr. 20780.

In theory, its main purpose is not revenue generation for the State, but to alter negative externalities such as damage to health and environmental pollution.
\end{abstract}

* Este trabajo tiene su origen en la tesis de Francisca Riffo y Pablo Torres, realizada el 2015 en la carrera de Derecho de la Universidad Católica de Temuco, para obtener la Licenciatura en Ciencias Jurídicas y guiada por la profesora PATriCia Toledo.

** Licenciada en Ciencias Jurídicas y Sociales, Universidad Católica de Temuco. Doctora en Derecho por la Universidad Pompeu Fabra, Barcelona. Profesora de Derecho Tributario, Universidad Austral de Chile. Correo electrónico: patricia.toledo@uach.cl

*** Licenciada en Ciencias Jurídicas y Sociales, Universidad Católica de Temuco. Correo electrónico: francisca.riffoaguilar@gmail.com

**** Licenciado en Ciencias Jurídicas y Sociales, Universidad Católica de Temuco. Correo electrónico: pablotorrespedreros@gmail.com

Artículo recibido el 31 de agosto de 2016 y aceptado para su publicación en este número el 18 de enero de 2019. 
The purpose of this paper is to study the non-tax taxation scheme of the 2014's Tax Reform and to demonstrate that, despite its name, its purpose is, primarily, that of tax collection.

Corrective taxes - tributary reform 2014 - taxes

\section{INTRODUCCIÓN}

$\mathrm{E}$ 129 de septiembre de 2014 se publicó en el Diario Oficial la Ley $N^{\circ}$ 20.780, reforma tributaria que modifica el sistema de tributación de la renta e introduce ajustes en el sistema tributario, en adelante "Reforma Tributaria 2014".

Este trabajo tiene por objeto de estudio cuatro impuestos establecidos por la mencionada Ley $\mathrm{N}^{\circ}$ 20.780; que afectan las siguientes actividades: consumo de tabaco; consumo de alcohol; uso de ciertos vehículos; emisión al aire de material particulado.

Este grupo de impuestos ha sido tratado por el gobierno como impuestos extrafiscales $^{1}$; su finalidad principal sería modificar externalidades negativas, como el daño a la salud y la contaminación medioambiental.

Nuestro propósito es analizar los impuestos extrafiscales de la Reforma Tributaria 2014 y determinar en qué sentido y en qué grado estos impuestos son, efectivamente, extrafiscales. Metodológicamente, comenzaremos definiendo una red conceptual acerca de los impuestos recaudatorios y respecto de los impuestos extrafiscales, clasificando estos últimos en propios e impropios. Acto seguido aplicaremos estos conceptos a cada uno de los denominados "impuestos extrafiscales” de la Reforma Tributaria 2014 para determinar cuáles de ellos son extrafiscales propios, cuáles son extrafiscales impropios y cuáles - no obstante su denominación- tienen una finalidad principalmente recaudatoria.

\section{RED CONCEPTUAL}

Tributo se puede definir como una prestación económica, de carácter obligatorio, establecido por la ley cuya finalidad es el financiamiento de los gastos públicos ${ }^{2}$. Los tributos se clasifican en tasas, contribuciones especiales e impuestos.

${ }^{1}$ Así, por ejemplo, el Mensaje del Proyecto de la Ley $\mathrm{N}^{\circ} 20.780$ se refiere a estos impuestos como "Impuestos ligados al cuidado del medio ambiente" e "Impuestos correctivos". Biblioteca DEL Congreso Nacional (2014): "Historia de la Ley 20.780", pp. 15 y 16. Disponible en: http://www.bcn.cl/historiadelaley/ nc/historia-de-la-ley/4406/ [consulta: 30 de diciembre de 2016].

${ }^{2}$ En el mismo sentido, el Modelo de Código Tributario del CIAT: un enfoque basado en la experiencia iberoamericana, en su artículo 2 dispone: "Tributos son las prestaciones pecuniarias que el Estado exige en ejercicio de su potestad tributaria y como consecuencia de la realización del hecho imponible previsto en la ley, al que esta vincula el deber de contribuir, con el objeto de satisfacer necesidades públicas". CIAT. Centro Interamericano de Administraciones Tributarias (2015). "Modelo de Código Tributario del CIAT: un enfoque basado en la experiencia Iberoamericana. 2015”. Disponible en: https://ciatorg-public.sharepoint. 
La recaudación de los ingresos provenientes de actividades gravadas con impuestos tiene por objetivo principal permitir que el Estado financie su Política Fiscal y cumplir así con el mandato constitucional de promover el bien común; esta finalidad se denomina función fiscal del Derecho Tributario.

En materia tributaria, hay quienes se oponen a la posibilidad de utilizar el Derecho Tributario para conseguir fines extrafiscales postulando que aquel estaría regido por un principio de neutralidad o no intervencionismo que implica que los tributos no deben influir en la adopción de decisiones económicas por parte de las personas afectadas por ellos ${ }^{3}$.

Actualmente existen varias legislaciones que permiten expresamente que el Derecho Tributario pueda cumplir funciones sociales y funciones económicas, distintas de la mera función fiscal, surge así el concepto de función extrafiscal del Derecho Tributario ${ }^{4}$.

Por definición, todo tributo tiene una finalidad recaudatoria; por tanto, clasificar un impuesto de fiscal o extrafiscal resulta, más bien, una cuestión de énfasis. Por ello, parece más adecuado hablar de impuestos con finalidad predominantemente fiscal e impuestos con finalidad predominantemente extrafiscal.

Siguiendo las ideas de Varona ${ }^{5}$, es posible afirmar que los elementos definidores de los tributos extrafiscales son los siguientes: perseguir con claridad una finalidad no recaudatoria que debe deducirse del articulado de su ley reguladora; la estructura interna del tributo debe impregnarse de los aspectos extrafiscales de tal forma que no advengan al gravamen como si de un añadido se tratara; y el tributo debe fomentar las conductas u operaciones acordes con la finalidad extrafiscal perseguida.

En virtud de lo anterior, es que se debe tener claro que la fiscalidad y la extrafiscalidad no necesariamente deben estar separadas la una de la otra. Existen impuestos con una finalidad fiscal claramente definida pero que incluso así pueden tener presente ciertos fines extrafiscales. Esto se logra comprender de mejor manera al clasificar la extrafiscalidad, atendiendo a sus distintos grados de intensidad, existiendo así impuestos extrafiscales propios e impuestos extrafiscales impropios.

Los impuestos extrafiscales propios son aquellos que en su fundamento y estructura están concebidos para lograr un objetivo de carácter extrafiscal, siendo esta finalidad

com/biblioteca/DocumentosTecnicos/Espanol/2015_Modelo_Codigo_Tributario_CIAT.pdf [consulta: 30 de diciembre de 2016].

3 A modo de ejemplo, ver: Zavala, J.L. Manual de Derecho Tributario. Editorial Jurídica Conosur: Santiago, 2001, pp. 18 y 19.

${ }^{4}$ A modo de ejemplo, en el ordenamiento jurídico español, el artículo 2.1 de la Ley 58/2003, de 17 de diciembre, General Tributaria, dispone: "1. Los tributos son los ingresos públicos que consisten en prestaciones pecuniarias exigidas por una Administración pública como consecuencia de la realización del supuesto de hecho al que la ley vincula el deber de contribuir, con el fin primordial de obtener los ingresos necesarios para el sostenimiento de los gastos públicos. Los tributos, además de ser medios para obtener los recursos necesarios para el sostenimiento de los gastos públicos, podrán servir como instrumentos de la política económica general y atender a la realización de los principios y fines contenidos en la Constitución". El destacado es nuestro.

${ }^{5}$ Varona, J., Extrafiscalidad y dogmática tributaria. Marcial Pons, Madrid, 2009, pp. 28 y ss. 
extrafiscal de tal relevancia para el impuesto, que condiciona su propia configuración y articula los elementos cuantitativos para lograr cumplir con aquellos fines.

Los impuestos extrafiscales impropios poseen una finalidad primordialmente fiscal, pero que puede incluir algunos incentivos o beneficios de naturaleza extrafiscal, que no lo alejan de su finalidad fundamentalmente recaudatoria, la que no se ve sustituida ni cuestionada por aquellos beneficios.

\section{IMPUESTO QUE AFECTA AL TABACO}

\section{Regulación legal}

En Chile esta materia se regula en el DL No 828 del Ministerio de Hacienda, que establece normas para el cultivo, elaboración, comercialización e impuestos que afectan al tabaco, publicado en 1974.

El cuerpo legal, antes citado, ha sido objeto de diversas modificaciones durante los últimos años. En esta ocasión solo se hará referencia a las que dicen relación con las variaciones que ha sufrido el impuesto al tabaco, regulado en el artículo 4 del DL No 828.

Al respecto, la legislación chilena contempla las siguientes tres leyes: a) Ley $\mathrm{N}^{\circ}$ 20.455, publicada el 31 de julio de 2010, en su artículo $6 \mathrm{~N}^{\circ} 2^{6}$; b) Ley $\mathrm{N}^{\circ} 20.630$, publicada el 27 de septiembre de 2012, en su artículo 67; c) Ley $\mathrm{N}^{\circ} 20.780$, de 29 de septiembre de 2014, en su artículo 4 letra $\mathrm{a}^{8}$. Todas ellas aumentan el impuesto específico y disminuyen el impuesto ad valorem ${ }^{9}$.

${ }^{6}$ Artículo $6^{\circ}$.- Introdúzcanse las siguientes modificaciones en el Decreto Ley $N^{\circ}$ 828, de 1974 , que establece normas para el cultivo, elaboración, comercialización e impuestos que afectan al tabaco: 2. Sustitúyase el artículo $4^{\circ}$ por el siguiente: "Artículo $4^{\circ}$.- Los paquetes, cajas o envoltorios de cigarrillos pagarán un impuesto específico equivalente a 0,0000675 unidades tributarias mensuales por cada cigarrillo que contengan; y, además, un impuesto de 62,3\%, que se aplicará sobre el precio de venta al consumidor, incluido impuestos, por cada paquete, caja o envoltorio, considerándose como entero toda fracción del impuesto inferior a un peso. Para estos efectos, el impuesto específico deberá calcularse tomando como base la unidad tributaria mensual vigente al momento de la determinación del impuesto".

${ }^{7}$ Artículo $6^{\circ}$.- Sustitúyase en el inciso primero, del artículo $4^{\circ}$, del Decreto Ley $\mathrm{N}^{\circ} 828$, de 1974 , que establece normas para el cultivo, elaboración, comercialización e impuestos que afectan al tabaco, el guarismo “0,0000675" y el porcentaje “62,3\%”, por "0,000128803” y “60,5\%”, respectivamente.

${ }^{8}$ Artículo $4^{\circ}$.- Introdúzcanse en el Decreto Ley $N^{\circ} 828$, del Ministerio de Hacienda, de 1974, que establece normas para el cultivo, elaboración, comercialización e impuestos que afectan al tabaco, las siguientes modificaciones:

a) Reemplácense, en el inciso primero del artículo $4^{\circ}$, los guarismos "0,000128803" y "60,5” por “0,0010304240” y “30”, respectivamente”.

${ }^{9}$ De acuerdo con el Sistema de Información sobre Comercio Exterior (SICE) un arancel específico es aquel "que se impone en término de cargas o cobros mometarios específicos por unidad o cantidad de mercancía importada"; en tanto que un arancel ad-valorem es aquel que se impone en términos de porcentaje sobre el valor de la mercancía. Sistema de Información sobre Comercio Exterior. SICE. Diccionario de término de comercio. Disponible en http://www.sice.oas.org/dictionary/TNTM_s.asp [consulta: 30 de diciembre de 2016]. 
Además se encuentra la Circular N ${ }^{\circ}$ 50, publicada el 3 de octubre del 2014 por el Servicio de Impuestos Internos - en adelante SII- que interpreta, con carácter obligatorio para los funcionarios del SII, los cambios introducidos por el mencionado artículo 4 de la Ley $\mathrm{N}^{\mathrm{0}} 20.780^{10}$.

Los trabajos legislativos de la Reforma Tributaria 2014 se retrotraen al 1 de abril del 2014, día en que el Poder Ejecutivo envía el Mensaje con el que se inicia la tramitación ${ }^{11}$-en adelante "El Proyecto"-.

En el Proyecto no se contemplaba modificación alguna al DL No $828^{12}$. La propuesta de incorporar el aumento de impuesto al tabaco se debe a la intervención de los parlamentarios Walker y Auth, en representación de los productores pisqueros y vitivinícolas, quienes con el alto impuesto al alcohol que proponía el gobierno podrían ver afectada su producción y así peligrar sus pequeños y medianos emprendimientos ${ }^{13}$. Por esta razón, proponen al ministro de Hacienda en ejercicio que presente una indicación que aumente el impuesto al tabaco, que compense la baja en la recaudación que, originalmente, se pretendía obtener con el impuesto al alcohol. El oficio con las indicaciones es presentado por el gobierno e incluye la propuesta de los parlamentarios en el artículo 2 bis del Proyecto.

Incluida la indicación al Proyecto, la discusión legislativa toma dos caminos. El primero - con una eminente función recaudatoria y justificada en lo perjudicial que podría resultar la Reforma para los pequeños y medianos productores vitivinícolas y pisquerosen ningún momento deja de sostener que lo que aquí importa es lograr reunir el dinero necesario para dar cumplimiento a las reformas del gobierno. El segundo, planteado por el ministro de Hacienda, quien señaló que se están siguiendo las buenas prácticas de la Organización para la Cooperación y el Desarrollo Económico -en adelante OCDEavanzando en impuestos correctivos ${ }^{14}$, cuestión apoyada por diversos parlamentarios.

Si bien en la discusión en el Congreso no se hizo referencia a la extrafiscalidad, con lo planteado por el ministro en ejercicio se comienza a considerar la idea de que el impuesto al tabaco se configure como un impuesto extrafiscal.

El resultado final de la Reforma Tributaria 2014 en cuanto al tabaco -siguiendo el mismo criterio seguido cuando se ha reformado este impuesto- es que se aumenta el impuesto específico y se disminuye el impuesto ad valorem; el primero aumentó ocho veces; el segundo, disminuyó $30 \%$.

${ }^{10}$ Servicio de Impuestos Internos (2014): “Circular 50”. Disponible en: http://www.sii.cl/documentos/ circulares/2014/circu50.pdf [consulta: 30 de agosto de 2016].

${ }^{11}$ Biblioteca del Congreso Nacional (2014): “Historia de la Ley No 20.780”. Disponible en: http:// www.bcn.cl/historiadelaley/nc/historia-de-la-ley/4406/ [consulta: 30 de agosto de 2016].

12 Biblioteca del Congreso Nacional (2014): "Historia de la Ley $\mathrm{N}^{\circ} 20.780$ ". Disponible en: http:// www.bcn.cl/historiadelaley/nc/historia-de-la-ley/4406/ [consulta: 30 de agosto de 2016], pp. 22 y 168.

${ }^{13}$ Biblioteca del Congreso Nacional (2014): "Historia de la Ley $\mathrm{N}^{\circ}$ 20.780". Disponible en: http:// www.bcn.cl/historiadelaley/nc/historia-de-la-ley/4406/ [consulta: 30 de agosto de 2016], p. 251.

${ }^{14}$ Biblioteca del Congreso Nacional (2014): "Historia de la Ley 20.780". Disponible en:http://www. bcn.cl/historiadelaley/nc/historia-de-la-ley/4406/ [consulta: 30 de agosto de 2016]. 


\section{Recaudación de impuestos y estadísticas relativas al tabaco}

Los datos que se utilizarán comprende entre 2011 y 2013; aun no existen datos oficiales de lo recaudado por concepto de impuesto al tabaco, posteriores a la entrada en vigencia de la Ley $\mathrm{N}^{\circ} 20.780$.

Desde el cuarto trimestre del 2011 hasta el tercer trimestre del 2013 la recaudación por concepto de impuesto específico al tabaco no ha ido constantemente al alza; sin perjuicio de ello, la variación del último trimestre en análisis sufrió un aumento del 28,5\% en comparación a igual trimestre del 2012, acumulándose un saldo de $\mathrm{MM} \$$ 42.887. En cuanto a la variación acumulada en los últimos 12 meses, esta alcanza $8,7 \%{ }^{15}$.

En seis años el impuesto al tabaco se ha aumentado tres veces, por lo que resulta esencial analizar la modificación tributaria que entró en vigencia el primer trimestre del 2013 en relación con el consumo de cigarrillos, el que alcanzó una cifra histórica al llegar a los 4.704.575.872 unidades de cigarrillos vendidos ${ }^{16}$.

Lo anterior explica el notorio aumento que registró la recaudación durante el primer trimestre del 2013, en el que se consolida el estancamiento del consumo de cigarrillos que se venía produciendo en la última década según el Undécimo Estudio Nacional de Drogas en Población General de Chile 2014 del SENDA -Servicio Nacional para la prevención y rehabilitación del consumo de drogas y alcohol-demuestra el aumento que se viene experimentando desde el 2012 y que en el 2014 se consolida con un aumento significativo de la prevalencia de consumo alguna vez en la vida; en 2012 era de 57,9\%; en 2014 alcanza a $64,4 \%{ }^{17}$ y se consumen 929,55 cigarrillos por persona al $2014^{18}$.

Las cifras expresadas coinciden con la tendencia histórica de aumento en la recaudación por impuesto al tabaco; salvo contadas excepciones, la constante siempre ha ido al alza, cuestión que se constata desde el año $1987^{19}$. Un último dato que resulta importante aportar es el que contiene la citada Circular $\mathrm{N}^{\circ} 50$, la que por medio de un ejemplo esclarece el valor total del impuesto en una cajetilla de 20 cigarrillos, este sería $\$ 1.474^{20}$.

15 Servicio de Impuestos Internos (2013): "Seguimientos de Impuestos: Período 211-2013”, p. 26. Disponible en: http://www.sii.cl/estadisticas/S_Impuestos_2013_III.pdf [consulta: 30 de agosto de 2016].

16 Servicio de Impuestos Internos (2013): "Seguimientos de Impuestos: Período 211-2013”, p. 27. Disponible en: http://www.sii.cl/estadisticas/S_Impuestos_2013_III.pdf [consulta: 30 de agosto de 2016].

17 Senda (2014): "Décimo Primer Estudio Nacional de Drogas en Población General de Chile", p. 21. Disponible en: http://www.senda.gob.cl/media/estudios/PG/2014_EstudioDrogas_Poblacion_General. pdf [consulta: 30 de agosto de 2016].

${ }^{18}$ Información disponible en: http://www.tobaccoatlas.org/topic/cigarette-use-globally/ [consulta: 30 de agosto de 2016].

${ }^{19}$ YañeZ, J., "Tabaquismo y tributación”, en Revista de Estudios Tributarios, Centro de Estudios Tributarios de la Universidad de Chile, N 3/2011, marzo 2011, p. 222. Disponible en: http://www.cetuchile.cl/revista/ revista_estudios_tributarios_3.pdf [consulta: 30 de julio de 2016].

${ }^{20}$ Servicio de Impuestos Internos (2014): "Circular 50", p. 2. Disponible en: http://www.sii.cl/ documentos/circulares/2014/circu50.pdf [consulta: 30 de agosto de 2016]. 


\section{Análisis crítico}

Con los antecedentes y datos recopilados se puede iniciar el análisis del tributo en cuestión.

Un impuesto extrafiscal debería cumplir con los tres postulados antes mencionados: el primero y más importante, pues de este proviene su denominación, es que debe perseguir una finalidad no fiscal; el segundo dice relación con la estructura interna del tributo; y el tercero se vincula con el fomento de conductas acordes a la finalidad extrafiscal ${ }^{21}$.

En cuanto al primer postulado, perseguir una finalidad no recaudatoria es un elemento que se debe analizar en virtud de todos los datos recopilados, por tanto, teniendo en cuenta que Chile es un país de fumadores (929,55 cigarrillos per cápita anuales), que el impuesto se ha aumentado constantemente y que la recaudación no ha disminuido, se podría inferir que el impuesto no logra ser extrafiscal.

Mediante este impuesto se logra recaudar cada vez más dinero, pero las externalidades negativas (alto consumo de cigarrillo per cápita) no disminuyen. Por tanto, este impuesto se puede calificar como un impuesto principalmente fiscal.

Se llega a esta conclusión en virtud de que las últimas tres reformas tributarias que han aumentado su impuesto tenían por fin último recaudar, ya sea para reconstruir el país o para financiar reformas estructurales que requieren de importantes cantidades de recursos. Sin embargo, cuando la Reforma Tributaria 2014 ingresó al Congreso Nacional, no se consideraba aumentar el impuesto al tabaco, surgiendo como alternativa a otros impuestos que se proyectaban como perjudiciales para ciertos sectores productivos, permitiendo de esta manera alcanzar los niveles de recaudación que se requerían. Por tanto, lo que estuvo presente fue el fin eminentemente fiscal.

En cuanto a la recaudación, un impuesto extrafiscal debiese tender después de un tiempo a reducir esta, porque se debería cumplir con el fin para el cual fue creado. En este caso, la recaudación desde 1987 a la fecha ha ido en constante alza y las Reformas que han aumentado la tributación no han logrado modificar la conducta de las personas; por tanto, se ha transformado en un tributo que aporta grandes sumas de dinero a las arcas fiscales.

No parece correcta la forma en que se estableció este impuesto considerando que el consumo de cigarrillos se relaciona con una dependencia química, se habla de una adicción; por tanto, su consumo está vinculado a un problema de salud pública, que no se soluciona con más o menos impuestos. Quien padece de dependencia a esta droga, no amortigua la adquisición del producto por el cambio de valor que en el mercado se transan los cigarrillos.

El segundo postulado introduce el análisis en la estructura interna del tributo, la que debe estar impregnada del carácter extrafiscal, no basta que sea un añadido como señala el profesor Varona. El problema en este punto radica en la simpleza del artículo 4 del DL N 828 y en lo escuetas que han sido sus modificaciones en los años 2010, 2012

${ }^{21}$ Varona, J., Extrafiscalidad y dogmática tributaria. Marcial Pons, Madrid, 2009, p. 163. 
y 2014, las que se han centrado en los guarismos que consagran el impuesto específico $\mathrm{y}$ el ad valorem.

En cuanto al tercer postulado, en Chile desde hace ya varios años se fomenta el no consumo de tabaco, esto en virtud de los efectos negativos que produce. Es así que existen variadas leyes que han buscado desincentivar su consumo y proteger a quienes no fuman; sin perjuicio de ello, en la ley en estudio, por tratarse de una legislación eminentemente tributaria, no existe más que la sola alza del impuesto como manera de fomentar el no consumo de cigarrillos. Considerando que Chile sigue siendo uno de los países que más tabaco consume en el mundo, surge la incertidumbre de si las medidas adoptadas son las suficientes para combatir el consumo de esta droga blanda.

\section{Impuesto QUE AFECTA A LAS BEBIDAS ALCOHÓLICAS}

\section{Regulación legal}

En Chile, el impuesto adicional que afecta a las bebidas alcohólicas se establece y regula en el artículo 42 del DL No 825 del Ministerio de Hacienda, ley sobre impuesto a las ventas y servicios, publicado el 31 de diciembre de 1974.

Dicha norma legal fue modificada por el artículo $2 \mathrm{~N}^{\circ} 10$ letras c) y d) de la Ley $\mathrm{N}^{\mathrm{o}} 20.780^{22}$, en la que se ordena aumentar el impuesto que afecta a las bebidas alcohólicas. En virtud de esta modificación, el SII dicta el día 3 de octubre de 2014 la Circular $\mathrm{N}^{\circ}$ 51, en la que se imparten instrucciones relativas a los cambios de tasa del impuesto a las bebidas alcohólicas ${ }^{23}$.

En la Historia de la Ley $\mathrm{N}^{\circ} 20.780$, consta que desde el inicio del Proyecto se consideró aumentar el impuesto a las bebidas alcohólicas, encuadrándolo dentro de los de tipo correctivo. Dicho impuesto operaría del siguiente modo: se aplica una tasa de $18 \%$ por el hecho de que una bebida contenga alcohol y se incrementa la tasa ad valorem de acuerdo con el contenido de alcohol a razón de $0,5 \%$ por cada grado de alcohol. Adicionalmente, se introduce una sobretasa de beneficio fiscal de 0,03 UTM por cada litro de alcohol puro que contengan dichas bebidas o la proporción correspondiente ${ }^{24}$.

${ }^{22}$ Artículo $2^{\circ}$.- Introdúcense las siguientes modificaciones en el Decreto Ley $\mathrm{N}^{\mathrm{o}}$ 825, de 1974, sobre Impuesto a las Ventas y Servicios: 10. En el artículo 42, reemplázase su inciso primero por el siguiente: c) Licores, piscos, whisky, aguardientes y destilados, incluyendo los vinos licorosos o aromatizados similares al vermouth, tasa del 31,5\%. d) Vinos destinados al consumo, comprendidos los vinos gasificados, los espumosos o champaña, los generosos o asoleados, chichas y sidras destinadas al consumo, cualquiera que sea su envase, cervezas y otras bebidas alcohólicas, cualquiera que sea su tipo, calidad o denominación, tasa del 20,5\%".

${ }^{23}$ Servicio de Impuestos Internos (2014): “Circular No 51”. Disponible en: http://www.sii.cl/documentos/ circulares/2014/circu51.pdf [consulta: 30 de agosto de 2016].

${ }^{24}$ Biblioteca del Congreso Nacional (2014): "Historia de la Ley 20.780". Disponible en: http://www. bcn.cl/historiadelaley/nc/historia-de-la-ley/4406/ [consulta: 30 de agosto de 2016]. 
En un principio se consideró aplicar el mismo impuesto a todas las bebidas alcohólicas, cualquiera fuese su tipo, calidad o denominación ${ }^{25}$.

Una vez iniciadas las exposiciones y la discusión general, el ministro de Hacienda de aquel entonces señaló que en cuanto a los impuestos correctivos aplicados a las bebidas alcohólicas estos en Chile son bajos si se compara con los países miembros de la OCDE, por lo que se aumenta este impuesto, el que tendrá tanto un componente ad valorem como uno en proporción al volumen de alcohol puro incluido en cada bebida ${ }^{26}$.

Durante el intercambio de ideas en la tramitación de esta ley, se generan distintas posturas respecto de cuánto debe ser el aumento al impuesto a las bebidas alcohólicas; por una parte, encontramos a los parlamentarios Alonso y Alday, quienes manifiestan su preocupación por los cientos de productores pisqueros. Esta preocupación se fundó en que en el Proyecto de ley se sindicó la actividad productora de bebidas alcohólicas como una actividad económica que debe realizar un esfuerzo adicional para aportar más, pero no se ha tenido en cuenta que la industria pisquera no es la actividad económica que más reporta ganancias en este país. Además, se señala en favor de la industria pisquera, en razón de los litros de bebidas alcohólicas que se comercializan, que en el mercado nacional se venden 700 millones de litros de cerveza, 220 millones de litros de vino y solamente 36 millones de litros de pisco. A su vez, las exportaciones de la industria pisquera no alcanzan el $2 \%$ de su producción.

Por esta razón, como se señaló en párrafos anteriores, el diputado Auth propone que se afecte al tabaco, lo que sería una posible opción frente al tributo adicional al pisco, cuestión que es apoyada de forma inmediata por el diputado Walker ${ }^{27}$. Es por ello que durante la tramitación se opta, según lo que en su momento señaló el ministro de Hacienda en ejercicio, por eliminar la sobretasa de 3 UTM que establecía el Proyecto original por hectolitros de alcohol puro. Decisión que también se toma siguiendo las buenas prácticas de la OCDE, avanzando así en impuestos correctivos ${ }^{28}$.

Posterior a la mención del entonces ministro de Hacienda, otros parlamentarios como Robles y Schilling señalan y solicitan al Ejecutivo que se mantenga la propuesta inicial, indicando que lo que se busca al aumentar los impuestos al alcohol y al tabaco es que se propenda a corregir ciertos comportamientos sociales, ya que los impuestos correctivos son complementarios a los procesos educativos ${ }^{29}$.

Finalmente, en virtud de todas las discusiones y opiniones otorgadas por los parlamentarios y expertos, además de los informes y estadísticas dadas a conocer, se termina

${ }^{25}$ Biblioteca del Congreso Nacional (2014): "Historia de la Ley 20.780". Disponible en: http://www. bcn.cl/historiadelaley/nc/historia-de-la-ley/4406/ [consulta: 30 de agosto de 2016].

${ }^{26}$ Biblioteca del Congreso Nacional (2014): "Historia de la Ley 20.780". Disponible en: http://www. bcn.cl/historiadelaley/nc/historia-de-la-ley/4406/ [consulta: 30 de agosto de 2016], p. 201.

${ }^{27}$ Biblioteca del Congreso Nacional (2014): "Historia de la Ley 20.780". Disponible en: http://www. bcn.cl/historiadelaley/nc/historia-de-la-ley/4406/ [consulta: 30 de agosto de 2016], pp. 250 y 251.

${ }^{28}$ Biblioteca del Congreso Nacional (2014): "Historia de la Ley 20.780". Disponible en: http://www. bcn.cl/historiadelaley/nc/historia-de-la-ley/4406/ [consulta: 30 de agosto de 2016], p. 478.

${ }^{29}$ Biblioteca del Congreso Nacional (2014): "Historia de la Ley 20.780". Disponible en: http://www. bcn.cl/historiadelaley/nc/historia-de-la-ley/4406/ [consulta: 30 de agosto de 2016]. 
estableciendo un impuesto adicional del 31,5\% para licores, piscos, whisky, aguardientes y destilados, incluyendo los vinos licorosos o aromatizados similares al vermouth; y una tasa adicional de 20,5\% para vinos destinados al consumo, comprendidos los vinos gasificados, los espumosos o champaña, los generosos o asoleados, chichas y sidras destinadas al consumo, cualquiera que sea su envase, cervezas y otras bebidas alcohólicas, cualquiera que sea su tipo, calidad o denominación.

\section{Estadísticas relativas al alcobol}

El consumo de alcohol en Chile es en promedio de 8,6 litros por persona al año ${ }^{30}$.

A lo anterior se sumarían los datos que aporta el informe El Consumo de Alcohol en Chile: Situación Epidemiológica, el que utilizando datos de la última encuesta realizada por SENDA, señala que la prevalencia mes de consumo de alcohol asciende a 48,9\% de la población. Basados en este dato se estima que 4.801 .318 personas entre 12 y 64 años consumieron alcohol el último mes en nuestro país. Cuando se evalúa el consumo intenso o Binge Drinking ( 5 o más tragos en hombres y 4 o más en mujeres) se concluye que 2.097.615 personas declaran haber tenido a lo menos un episodio de embriaguez en el último mes, lo que corresponde a $43,6 \%$ de la población consumidora ${ }^{31}$.

A los datos ya mencionados se agrega la estadística relativa a los accidentes de tránsito causados por la ingesta de alcohol y los relativos a las muertes por cirrosis hepática, causada por el excesivo consumo. Estos son algunos de los efectos negativos causados por las bebidas alcohólicas y su conocimiento ayuda a comprender mejor el aumento experimentado por la tasa impositiva.

Respecto de los accidentes de tránsito causados por conductores bajo la influencia del alcohol, entre el 2011 y 2012 se observa una disminución que va desde los 840 a los 548 eventos donde existe presencia de alcohol. Los accidentes causados por conductores en estado de ebriedad, entre los mismos años, también registran un descenso que va desde los 4.206 en el 2011 a los 3.130 en $2012^{32}$. La OMS en el 2012 aporta estadísticas referente a la mortalidad que causa la cirrosis hepática provocada por la excesiva ingesta de alcohol, que alcanza 66,3\% del total de muertes en hombres y el $66,9 \%$ en mujeres ${ }^{33}$. El último antecedente a analizar es la comparación entre la tasa anterior a la Reforma y la que se aplica desde el 1 de octubre de 2014.

${ }^{30}$ Mellado, A., "Análisis del nuevo impuesto a los alcoboles", en Repositorio Académico de la Universidad de Chile, enero 2015, pp. 18-19. Disponible en: http://www.repositorio.uchile.cl/handle/2250/129680 [consulta: 30 de agosto de 2016].

${ }^{31}$ SENDA (2016): "El consumo de alcohol en Chile: Situación epidemiológica”, p. 4. Disponible en: http://www.senda.gob.cl/media/estudios/otrosSENDA/2016_Consumo_Alcohol_Chile.pdf [consulta: 30 de agosto de 2016].

32 Instituto Nacional de Estadísticas (2013): “Carabineros, Informe anual 2012”, p. 227. Disponible en: http://www.ine.cl/canales/menu/publicaciones/calendario_de_publicaciones/pdf/informe_anual_ carabineros_2012.pdf [consulta: 30 de agosto de 2016].

33 World Health Organization (2014). Disponible en: http://www.who.int/substance_abuse/ publications/global_alcohol_report/msb_gsr_2014_2.pdf?ua=1 [consulta: 30 de agosto de 2016]. 
Al observar los datos respectivos no se divisa mayor variación en ninguno de los casos, las bebidas fermentadas aumentan en $36,7 \%$ y los destilados $16,7 \%$, aumentos en sus tasas impositivas que al traspasarlo al precio que paga el consumidor no reflejan mayor variación ${ }^{34}$.

\section{Análisis crítico}

El consumo de alcohol en Chile se encuentra en niveles altos y genera externalidades negativas, entre las que se encuentran accidentes en vehículos motorizados a causa del consumo de alcohol, enfermedades que afectan la salud del bebedor adicto, los crímenes asociados al consumo de alcohol y el constante aumento en el gasto público a causa de las externalidades anteriores ${ }^{35}$.

Si se tiene en consideración todo esto, encuentra fundamento la intención del gobierno de Chile de aumentar el impuesto a este tipo de bebidas, aumento que estuvo presente desde un principio en la Reforma Tributaria 2014 y de manera mucho más gravosa de lo que finalmente se terminó aprobando.

Del análisis de la Historia de la Ley queda claro que la intención va más allá de la sola recaudación, y a lo largo de la discusión en el Congreso siempre estuvo presente la necesidad de disminuir el consumo, teniendo en consideración los datos aportados por la OMS y el propósito de alcanzar los niveles de la OCDE.

Otro argumento que se debe considerar es la elasticidad de la demanda, la que señala en qué medida varía la cantidad demandada a partir de un cambio en el precio, el que a su vez cambiaría debido a que varía la tasa impositiva ${ }^{36}$.

Por tanto, se ha planteado que a mayor precio por motivo del alza de impuestos, menor sea el consumo, cuestión que reflejaría una mayor elasticidad de la demanda. Siendo esto lo ideal en el caso concreto, resulta que la demanda por alcohol tiene componentes inelásticos, como son los hábitos sociales arraigados, el hecho que el alcohol no tiene sustitutos directos y el componente adictivo con el que cuenta ${ }^{37}$.

Lo anterior va a significar que ante el alza que experimentaron los alcoholes no se lograría modificar el hábito del consumo, debido a la baja incidencia que tuvo en

${ }^{34}$ Mellado, A., "Análisis del nuevo impuesto a los alcoboles", en Repositorio Académico de la Universidad de Chile, enero 2015, p. 31. Disponible en: http://www.repositorio.uchile.cl/handle/2250/129680_[consulta: 30 de agosto de 2016].

35 YaÑEZ, J., “Tabaquismo y tributación”, en Revista de Estudios Tributarios, Centro de Estudios Tributarios de la Universidad de Chile, No 3/2011, marzo 2011, p. 37. Disponible en: http://www.cetuchile. cl/revista/revista_estudios_tributarios_3.pdf [consulta: 30 de agosto de 2016].

${ }^{36}$ Mellado, A., "Análisis del nuevo impuesto a los alcoholes", en Repositorio Académico de la Universidad de Chile, enero 2015, p. 51. Disponible en: http://www.repositorio.uchile.cl/handle/2250/129680_[consulta: 30 de agosto de 2016].

${ }^{37}$ Mellado, A., "Análisis del nuevo impuesto a los alcoboles", en Repositorio Académico de la Universidad de Chile, enero 2015, p. 51. Disponible en: http://www.repositorio.uchile.cl/handle/2250/129680_[consulta: 30 de agosto de 2016], pp. 51-53. 
el precio final del producto la modificación de la tasa impositiva que se aplica a las bebidas alcohólicas.

En consecuencia, no se logra cumplir con el objetivo de un impuesto extrafiscal, el que tiene por principal función modificar la conducta que genera las externalidades negativas, y termina siendo un impuesto eminentemente recaudador, retomando la línea general de la Reforma Tributaria 2014.

En síntesis, este impuesto es extrafiscal impropio, debido a que se pretende hacer frente y erradicar las diversas externalidades negativas; sin embargo mantiene la intención recaudatoria.

\section{IMPUESTOS QUE AFECTAN A LOS VEHÍ́CULOS Y A LAS EMISIONES CONTAMINANTES}

\section{Regulación legal}

La Reforma Tributaria 2014 incluye dos nuevos tributos, conocidos coloquialmente como impuestos verdes, los que según la Historia de la Ley $\mathrm{N}^{\circ} 20.780$ cuentan con características extrafiscales, gravando por una parte a los vehículos nuevos, livianos y medianos, y, por otro lado, el que se aplica a las emisiones al aire de material particulado.

La inclusión de estos nuevos impuestos obedece a los magros resultados obtenidos al comparar Chile con los países de la OCDE, en cuanto a sus políticas tendientes a proteger el medio ambiente. Es por ello que surge la necesidad de alcanzar los parámetros establecidos por esta Organización, con el objetivo de propender y avanzar hacia un crecimiento verde.

Para lograr esta finalidad, se tienen en consideración cuatro objetivos primordiales planteados por la OCDE: establecer una economía con emisiones bajas de carbono y con eficiencia de recursos; mantener la base de activos naturales; mejorar la calidad de vida de las personas; y emprender acciones de políticas públicas adecuadas junto con concretar las oportunidades económicas que supone el crecimiento verde ${ }^{38}$.

La preocupación por regular estos impuestos ligados al medio ambiente se esboza en los objetivos del Proyecto de la Ley N ${ }^{\circ} 20.780$; sin embargo, lo planteado originalmente para cada uno de estos tributos dista del resultado final.

En cuanto a la normativa legal que regula el impuesto aplicado a los vehículos nuevos, livianos y medianos, se encuentran: a) los artículos 3 y décimo transitorio de la Ley $\mathrm{N}^{\circ}$ 20.780; b) el Decreto 241 del Ministerio de Transporte y Telecomunicaciones -en adelante MTT- publicado el 29 de noviembre de 2014; c) la Resolución Exenta del SII N 119 de 2014.

\footnotetext{
${ }^{38}$ Organisation for Economic Co-Operation and Development (2015): “¿En qué situación está Chile comparativamente?”. Disponible en: http://www.oecd360.org/oecd360/pdf/domain21 2sr2soko0d.pdf [consulta: 30 de agosto de 2016].
} 
En cuanto a la Ley $N^{\circ} 20.780$, en su artículo 3 se regula la creación del impuesto; la forma de calcularlo; se indican los vehículos que quedan exentos del pago del impuesto; se ordena al MTT determinar el rendimiento urbano y la cantidad de emisiones contaminantes que emiten los vehículos; se ordena al SII calcular el pago de dicho impuesto; y a la Tesorería General de República recibir el pago. En el artículo décimo transitorio se señala que 30 días después de publicado el Reglamento comenzará a regir la aplicación del impuesto de manera paulatina, ya que una de las cifras de esta fórmula irá aumentando en el tiempo de la siguiente manera: utilizándose desde la publicación del reglamento hasta diciembre del 2015, el guarismo 60; durante el 2016, el guarismo 90; y posteriormente alcanzará la cifra 120, como lo consagra el artículo 3 al estipular la fórmula.

En el Decreto 241 del MTT, publicado el 29 de noviembre de 2014, regula que la determinación del rendimiento urbano y las emisiones de NOx se realizará mediante el proceso de homologación, cuyo resultado final se encuentra en el sitio web de este Ministerio.

La Resolución Exenta del SII N 119 de 2014 regula el cálculo del pago del impuesto y mantiene en su página web el asistente de cálculo de impuesto a emisiones contaminantes de vehículos nuevos ${ }^{39}$, el que utiliza la fórmula que consagra la mencionada Ley $\mathrm{N}^{\circ} 20.780$.

Por otra parte, se destaca en la discusión parlamentaria. La regulación que se planteó en el Proyecto original de la Ley $\mathrm{N}^{\circ} 20.780$ dio un vuelco importante debido a que la carga tributaria con la que se pretendía gravar a los vehículos, en principio, se aplicaría a aquellos importados cuyo motor funcionara a diésel, impuesto que se pagaría al Servicio Nacional de Aduanas ${ }^{40}$. El resultado final fue gravar con el impuesto a todos los vehículos nuevos, livianos y medianos, que sean adquiridos dentro del país, sin importar el tipo de combustible que utilicen.

Lo anterior se explica por la preocupación que surgió en el Congreso Nacional al ver la posibilidad de que Chile faltara a obligaciones de orden internacional, como son las que constan en el General Agreement on Tariffs and Trade (GATT) ${ }^{41}$, esto respecto de la importación. En cuanto al tipo de combustible se generaba la discusión de cuál contamina más y no habiendo claridad al respecto y para evitar una posible diferenciación, que pudo ser considerada arbitraria, es que se optó por gravar de igual forma.

Por su parte, el impuesto a las emisiones de fuente fija se regula en el artículo 8 de la Ley $\mathrm{N}^{\circ} 20.780$, el que contempla la fórmula que se utilizará para su cálculo, en la que no se profundizará debido a que este nuevo tributo aún no comienza a regir, como se desprende del artículo transitorio decimocuarto, ya que este se aplicará a contar del 2017 y deberá ser pagado por primera vez el 2018. Es deber del Ministerio del Medio

\footnotetext{
${ }^{39}$ Información disponible en:https://www4.sii.cl/calcImpVehiculoNuevoInternet/internet.html [consulta: 30 de agosto de 2016].

${ }^{40}$ Biblioteca del Congreso Nacional (2014): "Historia de la Ley 20.780”. Disponible en: http://www. bcn.cl/historiadelaley/nc/historia-de-la-ley/4406/ [consulta: 30 de agosto de 2016].

${ }^{41}$ Biblioteca del Congreso Nacional (2014): “Historia de la Ley 20.780". Disponible en: http://www. bcn.cl/historiadelaley/nc/historia-de-la-ley/4406/ [consulta: 30 de agosto de 2016].
} 
Ambiente indicar mediante Decreto Supremo los establecimientos que se encuentran en la situación del inciso primero del artículo 8 y debe dictar el Reglamento que permitirá determinar los factores de emisión-concentración por comuna. La Superintendencia del Medio Ambiente es quien deberá certificar en marzo de cada año las emisiones efectuadas en el año calendario anterior y entregar al SII los antecedentes necesarios para el cálculo del impuesto, el que se deberá pagar en la Tesorería General de la República.

Es necesario señalar que no se tienen antecedentes preliminares que permitan una comparación en cuanto a la modificación que pueda sufrir la tributación, luego de la aplicación de la Reforma, y además se suma que para realizar el cálculo de la fórmula se requieren antecedentes que deben ser aportados por el Ministerio y la Superintendencia del Medio Ambiente.

Sin perjuicio de lo anterior, y para cerrar desde ya el análisis del impuesto a las emisiones contaminantes, es que se plantea que este impuesto cumple con las características necesarias para ser denominado, a priori, extrafiscal, pero se debe esperar hasta el 2018 y posteriores para determinar con certeza que el impuesto ha corregido las externalidades negativas y que los contribuyentes afectados han realizado las inversiones necesarias para estos efectos.

\section{Estadísticas}

Como se advirtió en los párrafos previos, los impuestos que se tratan en este apartado son tributos que se crearon recientemente, por tanto, a la fecha de redacción de este artículo no existen antecedentes oficiales que se puedan analizar. Por dicha razón es que se crea una tabla ejemplificadora, con la sola finalidad de dar a conocer un estimativo de cuánto sería el valor del impuesto a pagar por la compra de un vehículo nuevo, liviano y mediano.

Para la creación de dicha tabla se revisaron y utilizaron los datos entregados por el SII mediante su sitio web, en cuanto a la tasación fiscal, rendimiento urbano y emisiones contaminantes de los vehículos livianos ${ }^{42}$.

De los datos entregados en la tasación de los vehículos se utilizaron los siguientes ítems: marcas, modelos, precios y años. Por otra parte, de los datos que se entregan por medio del archivo que contiene las emisiones contaminantes, se utilizó el Código de Informe Técnico (CIT), esto con la finalidad de hacer coincidir los modelos de vehículos en ambas tablas.

Respecto de la realización del ejercicio antes señalado, se debe tener presente que al momento de llevarlo a cabo se determinó que no todos los modelos de vehículos que están tasados el 2015 por el SII se encuentran en la tabla que determina la cantidad de emisiones contaminantes de cada uno. Es por esta razón que la selección aleatoria de vehículos solo considera automóviles año 2014, para saber y dar a conocer cuánto sería lo que se pagaría por impuesto en la compra de un vehículo liviano.

42 Información Disponible en: http://www.sii.cl/pagina/actualizada/noticias/tasacion_vehiculos.htm [consulta: 30 de agosto de 2016 ]. 
Tabla ejemplificadora del valor del impuesto a los vehículos nuevos, livianos y medianos

\begin{tabular}{|c|c|c|c|c|c|c|}
\hline Marca & Modelo & Combustible & Valor & Emisiones NOx & $\begin{array}{l}\text { Impuesto } \\
\text { a Pagar }\end{array}$ & $\begin{array}{c}\text { Valor al } \\
17 / 05 / 15\end{array}$ \\
\hline Rolls-Royce & Phantom SWB & Bencina & $\$ 249.990 .000$ & $0,01870 \mathrm{Gr} / \mathrm{Km}$ & 136,14 UTM & $\$ 5.921 .954$ \\
\hline Ferrari & $\mathrm{FF}$ & Bencina & $\$ 134.320 .000$ & $0,01180 \mathrm{Gr} / \mathrm{Km}$ & 78,03 UTM & $\$ 3.394 .227$ \\
\hline Land Rover & Range Rover 4.4 Sd VB & Diésel & $\$ 67.160 .000$ & $0,13646 \mathrm{Gr} / \mathrm{Km}$ & 49,20 UTM & $\$ 2.140 .151$ \\
\hline Mercedes Benz & SI63Amg & Diésel & $\$ 64.730 .000$ & $0,03359 \mathrm{Gr} / \mathrm{Km}$. & 27,25 UTM & $\$ 1.185 .348$ \\
\hline BMW & $\begin{array}{l}\text { X5 Xdrive } 30 \text { D } \\
\text { Executive W Sro }\end{array}$ & Diésel & $\$ 26.830 .000$ & $0,13270 \mathrm{Gr} / \mathrm{Km}$ & 17,72 UTM & $\$ 770.802$ \\
\hline Land Rover & $\begin{array}{c}\text { Discovery } 43.0 \mathrm{~V} 6 \\
\text { Dsl Se }\end{array}$ & Diésel & $\$ 26.820 .000$ & $0,16114 \mathrm{Gr} / \mathrm{Km}$ & 21,89 UTM & $\$ 952.193$ \\
\hline Mercedes Benz & SLK350 (Nuevo) & Bencina & $\$ 26.150 .000$ & $0,00569 \mathrm{Gr} / \mathrm{Km}$. & 7,23 UTM & $\$ 314.498$ \\
\hline Land Rover & Discovery 43.0 V6 D. Se & Diésel & $\$ 26.150 .000$ & $0,16114 \mathrm{Gr} / \mathrm{Km}$ & 21,34 UTM & $\$ 928.269$ \\
\hline Dodge & Journey Se $2.41 \mathrm{At}$ & Bencina & $\$ 11.800 .000$ & $0,13342 \mathrm{Gr} / \mathrm{Km}$ & 7,80 UTM & $\$ 339.292$ \\
\hline
\end{tabular}

Esta tabla demuestra que efectivamente los vehículos que utilizan combustible de tipo diésel contaminan más que los que utilizan bencina, además se desprende de ella que el valor del impuesto está directamente relacionado con el valor del vehículo. Por tanto, el alza en el precio final no es significativa, porque los valores del impuesto no modifican la intención del comprador, el que si dispone del dinero para pagar un automóvil de cierta cantidad de millones, también dispondrá de lo necesario para pagar el tributo.

En el caso en que a una persona el valor del impuesto la prive de la posibilidad de adquirir determinado vehículo nuevo, recién ahí podría optar por un automóvil nuevo más barato, uno menos contaminante o simplemente por un vehículo usado, en virtud de su capacidad económica.

\section{Análisis crítico}

El impuesto a vehículos nuevos, livianos y medianos, merece ser criticado en virtud al quantum del impuesto, que al igual que en el gravamen a las emisiones contaminantes, se calcula a base de una fórmula expuesta en los apartados anteriores. En los distintos casos que se analizaron no se observa una gran diferencia para una persona que va a invertir una suma no menor de dinero y la que se calcula a mediano y largo plazo.

El impuesto en cuestión no representa un gran porcentaje del precio de venta y si bien es mayor en aquellos vehículos más contaminantes, podría suceder que se prefiera pagar el impuesto al momento de la compra y recuperarlo en el menor gasto de combustible que se generará a futuro por consumir diésel y no bencina. 
Son estas consideraciones las que llevan a concluir que existe una errónea aplicación del impuesto, ya que no logrará corregir las externalidades negativas, ya que solo busca aumentar la recaudación gravando bienes de consumo con un impuesto adicional.

Si el Estado tiene la necesidad de incentivar la compra de vehículos menos contaminantes y generar un cambio de conciencia real, se requieren medidas como que el impuesto sea aplicado a autos usados; no deberían excluirse de la aplicación del gravamen a los vehículos de transporte de pasajeros; y el impuesto debería pagarse en proporción a lo que contamina, una vez al año al momento de someterse al proceso de revisión técnica.

En síntesis, este impuesto podría ser considerado eminentemente fiscal. Pero es la naturaleza de los impuestos verdes, dentro de los que está incluido; y el espíritu del legislador, los que permiten que sea clasificado como extrafiscal impropio.

\section{Conclusiones}

La realización de este trabajo permite concluir lo siguiente:

1. En cuanto al impuesto que afecta al tabaco, se ha calificado como impuesto fiscal. Cuando la Reforma Tributaria 2014 ingresó al Congreso Nacional no se consideraba aumentar el impuesto al tabaco, surgiendo como alternativa a otros impuestos que se proyectaban como perjudiciales para ciertos sectores productivos, permitiendo de esta manera alcanzar los niveles de recaudación que se requerían; por tanto, lo que estuvo presente fue el fin eminentemente fiscal. Influye también en esta conclusión, el hecho de configurarse el tabaco como una droga blanda generadora de dependencia, lo que implica que las externalidades negativas no se resuelven simplemente con impuestos más gravosos.

2. En cuanto al impuesto que afecta a las bebidas alcohólicas, se ha considerado un impuesto extrafiscal impropio. El alcohol no tiene sustitutos directos, lo que transforma su demanda en inelástica; sin embargo, es posible que exista una variación entre el consumo de diversos tipos de bebidas alcohólicas o se consuman bebidas de menor calidad y por tanto de menor precio. A esto se suma que el precio final de consumo no sufre mayor variación que permita cambiar los hábitos arraigados en la sociedad chilena, esto queda claro en las cifras que se expusieron, las que demuestran que Chile es un país que consume grandes cantidades de alcohol.

3. Uno de los impuestos denominados verdes que se analizó corresponde al adicional que se aplica a los automóviles nuevos, livianos y medianos, el que tiene por objetivo desincentivar la adquisición y uso de los vehículos más contaminantes; para determinar esta característica la ley contempla la fórmula que permite calcularlo. Este impuesto adicional no es suficiente para erradicar la externalidad negativa, representada por la contaminación que genera el parque automotriz; esta crítica se basa en que el impuesto que se aplica no representa un mayor porcentaje del valor 
del automóvil, por tanto, quien va a comprar un vehículo nuevo es muy probable que también cuente con los recursos suficientes para hacerlo. Cambiaría esta situación si la ley considerara diferentes incentivos y no solo el mero gravamen que genera el tributo.

4. El impuesto que afecta a las emisiones al aire de material particulado, también considerado un impuesto verde; para su cálculo el legislador también debió crear una fórmula que permita su aplicación.

Este impuesto aún no comienza a regir y tampoco se cuenta con todos los datos que requiere la fórmula; por esta razón, no se incluyen datos estadísticos en el trabajo, ni siquiera a modo de ejemplo. Pese a la falta de antecedentes necesarios para concluir acerca de la extrafiscalidad de este impuesto, basta con la clara intención del legislador, lo oneroso de la actividad que se grava y la naturaleza del impuesto para señalar a priori, que se está frente a un impuesto extrafiscal.

\section{BIBLIOGRAFÍA}

Biblioteca del Congreso Nacional. "Historia de la Ley 20.780". Disponible en: http://www. bcn.cl/historiadelaley/nc/historia-de-la-ley/4406/ [consulta: 30 de diciembre de 2016].

Centro Interamericano de Administraciones Tributarias. "Modelo de Código Tributario del CIAT: un enfoque basado en la experiencia Iberoamericana. 2015”. Disponible en: https:// ciatorg-public.sharepoint.com/biblioteca/DocumentosTecnicos/Espanol/2015_Modelo_ Codigo_Tributario_CIAT.pdf [consulta: 30 de diciembre de 2016].

Instituto Nacional de Estadísticas. Informe anual 2012. Disponible en: http://www.ine. $\mathrm{cl} /$ canales/menu/publicaciones/calendario_de_publicaciones/pdf/informe_anual_carabineros_2012.pdf [consulta: 30 de agosto de 2016].

Mellado, Andrés. Análisis del nuevo impuesto a los alcoboles. Disponible en: http://repositorio. uchile.cl/handle/2250/1296808 [consulta: 30 de agosto de 2016].

Organisation for Economic Cooperation and Development. ¿En qué situación está Chile comparativamente? Disponible en: http://www.oecd360.org/oecd360/pdf/domain21 media1988__3104882sr2soko0d.pdf [consulta: 30 de agosto de 2016].

Servicio de Impuestos Internos. Asistente de Cálculo de Impuesto a Emisiones Contaminantes de Vehículos Nuevos. Disponible en: https://www4.sii.cl/calcImpVehiculoNuevoInternet/internet. html [consulta: 30 de agosto de 2016].

Servicio de Impuestos Internos. Circular $N^{o} 50$ de 2014. Disponible en: http://www.sii.cl/ documentos/circulares/2014/circu50.pdf [consulta: 30 de agosto de 2016].

Servicio de Impuestos Internos. Circular $N^{o} 51$ de 2014. Disponible en: http://www.sii.cl/ documentos/circulares/2014/circu51.pdf [consulta: 30 de agosto de 2016].

Servicio De Impuestos Internos. Resolución Exenta $N^{\circ} 119$ de 2014. Disponible en: http:// www.sii.cl/documentos/resoluciones/2014/reso119.pdf [consulta: 30 de agosto de 2016].

Servicio de Impuestos Internos. Seguimiento de impuestos. Periodo 2011-2013. Disponible en: http://www.sii.cl/estadisticas/S_Impuestos_2013_III.pdf [consulta: 30 de agosto de 2016].

Varona, Juan. Extrafiscalidad y dogmática tributaria. Madrid de España: Marcial Pons, 2009. 163 p.

World Health Organization. Country Profiles. Disponible en: http://www.who.int/ substance_abuse/publications/global_alcohol_report/msb_gsr_2014_2.pdf?ua =1 [consulta: 30 de agosto de 2016]. 
YaÑEz, José. Impuestos Indirectos en Chile. Disponible en: http://www.cepchile.cl/dms/archivo_5008_3149/doc_Yanez_Impuestos-indirectos-en-Chile.pdf [consulta: 30 de agosto de 2016].

YaÑEZ, José. Tributación y Tabaquismo. Disponible en: http://www.cetuchile.cl/revista/revista_estudios_tributarios_3.pdf [consulta: 30 de agosto de 2016]. 